\title{
A Influência da Idade e da Escolaridade nas Praxias Construtivas do Teste NEUPSILIN
}

\author{
Maila Rossato Holz, Renata Kochhann \\ Pontifícia Universidade Católica do Rio Grande do Sul, Porto Alegre-RS, Brasil \\ Jerusa Fumagalli de Salles \\ Universidade Federal do Rio Grande do Sul, Porto Alegre-RS, Brasil \\ Maria Alice de Mattos Pimenta \\ Universidade Federal do ABC, Santo André-SP, Brasil \\ Rochele Paz Fonseca ${ }^{1}$ \\ Pontifícia Universidade Católica do Rio Grande do Sul, Porto Alegre-RS, Brasil
}

\section{RESUMO}

Avaliação das praxias construtivas, embora tradicional, tem sido insuficientemente explorada no exame neurocognitivo. O objetivo foi verificar efeito de idade e escolaridade, assim como interação entre os itens de praxias construtivas no Instrumento de Avaliação Neuropsicológica Breve - NEUPSILIN, obtendo-se normas de referência por desempenho. Participaram 613 adultos e idosos entre 19 até 90 anos. Realizou-se quatro tarefas, três de cópia (quadrado, margarida e cubo) e uma produção espontânea (relógio). Conduziu-se MANCOVA para avaliar efeito da idade e escolaridade nos grupos; post hoc Bonferroni para normas nos desenhos. Houve efeito de idade no quadrado e no relógio e efeito de escolaridade no relógio. Ocorreu interação entre idade e escolaridade no cubo e na margarida em que o desempenho aumentou nos grupos de maior escolaridade e diminuíram com aumento da idade. Percebe-se a influência da idade e escolaridade como variáveis relevantes para dados normativos de desempenho em praxias construtivas.

Palavras-chave: praxias construtivas, NEUPSILIN, idade, escolaridade, normas.

\section{ABSTRACT - The Influence of Age and Schooling in the Constructive Praxis of the NEUPSILIN Test}

Constructive praxis evaluation, although traditional, has been insufficiently explored in the neurocognitive examination. The objective of this study was to verify the effect of age and schooling, as well as interaction between the items of constructive praxis in the Brief Neuropsychological Assessment Instrument (NEUPSILIN), obtaining performance benchmarks. A total of 613 adults and elderly between 19 and 90 years of age participated. There were four tasks: three copying (square, daisy and cube) and one spontaneous production (clock). MANCOVA was conducted to evaluate effects of age and schooling in the groups; post hoc Bonferroni for standards in drawings. Age had an effect on the drawings of the square and the clock, schooling had an effect on the drawings of the clock. There was interaction between age and schooling in the cube and daisy drawings, in which performance increased in the groups with higher schooling and decreased with increasing age. We can see the influence of age and schooling as relevant variables for normative performance data in constructive praxis.

Keywords: constructive praxis, NEUPSILIN, age, education, standards.

\section{RESUMEN - Influencia de la Edad y la Escolaridad en las Praxias Constructivas del Test NEUPSILIN}

La evaluación de las praxias constructivas, aunque tradicional, no ha sido suficientemente investigada en el examen neurocognitivo. El objetivo fue verificar la interacción entre edad y escolaridad, así como la interacción entre los ítems de praxias constructivas en el Instrumento de Evaluación Neuropsicológica Breve - NEUPSILIN, obteniéndose normas de referencia por rendimiento. Participaron 613 adultos y ancianos con edades entre 19 y 90 años. Fueron realizadas cuatro tareas, tres copias (cuadrado, margarita y cubo) y una producción espontánea (reloj). Se llevó a cabo MANCOVA para evaluar el efecto de edad y escolaridad en los grupos; post hoc Bonferroni para las normas en los dibujos. Hubo efecto de edad en el cuadrado y en el reloj; también hubo efecto de escolaridad en el reloj. Hubo interacción entre edad y escolaridad en el cubo y la margarita donde el rendimiento aumentó en los grupos con más escolaridad y disminuyó en los grupos de edad más avanzada. La influencia de edad y escolaridad parecen ser variables relevantes para datos normativos de rendimiento en praxias constructivas.

Palabras clave: praxias constructivas, NEUPSILIN, edad, escolaridad, normas. 
As praxias construtivas $(\mathrm{PC})$ se referem à capacidade em integrar partes representativas de um determinado símbolo/objeto para avaliar suas habilidades motoras, visuoespaciais e visuoconstrutivas (Hubbard et al., 2008; Santana, Duro, Freitas, Alves, \& Simões, 2013). Testes para a avaliação das habilidades visuoconstrutivas são normalmente utilizados como rastreio cognitivo devido a sua fácil aplicação e rápida execução (Hubbard et al., 2008), além de permitir a avaliação de componentes, como habilidades motoras, dimensões espaciais, praxias e funções executivas (Aprahamian, Martinelli, Cecato, Izbicki, \& Yassuda, 2011). Assim percebe-se que o uso de tarefas de PC é muito comum em quadros neurológicos, psiquiátricos, ou em rastreios clínicos cognitivos, por elas darem uma noção global sobre múltiplas habilidades cognitivas de maneira rápida. Com isso, o uso em pacientes com quadros neurodegenerativos do envelhecimento nas habilidades visuoconstrutivas podem estar prejudicadas (Freeman et al., 2000; Parsey \& Schmitter-Edgecombe, 2011), já em pacientes deprimidos não foram encontradas diferenças significativas (Rosenstein, 1999).

As figuras mais utilizadas na literatura para avaliar habilidades de PC nos diferentes transtornos são o desenho do relógio (Parsey \& Schmitter-Edgecombe, 2011), a Figura Complexa Rey-Osterrieth (ElderkinThompson et al., 2004; Rosenstein, 1999), teste de Retenção Visual de Benton - BVRT (Salles, Bandeira, Trentini, Segabinazi, \& Hutz, 2015; Segabinazi, Duarte Junior, Bandeira, Trentini, Hutz, \& Salles, 2013), Teste da Figura Complexa de Taylor - versão simplificada (Taylor, 1969) e o cubo (Fillenbaum et al., 2005; Robbins et al., 2013; Rossetti, Lacritz, Cullum, \& Weiner, 2011). Tarefas com o uso de desenho para avaliar habilidades visuoconstrutivas parecem altamente relacionadas com a gravidade da demência de Alzheimer (Gragnaniello, Kessler, Bley, \& Mielke, 1998), pois elas permitem a manipulação de um conhecimento visuoespacial anterior $\mathrm{e}$ a criação visuomotora desse desenho (Gaestel, Amieva, Letenneur, Dartigues, \& Fabrigoule 2006). Entretanto, o uso da flor (Kumral, EvAJpan, Sirin, \& Alpaydin, 2012) e do quadrado (Rodrigues, Pawlowski, Zibetti, Fonseca, \& Parente, 2011) ainda são menos explorados na literatura, frente ao uso do cubo e do relógio para avaliar habilidades visuoconstrutivas e de praxias motoras. Entretanto, é a partir de itens mais simples que é possível identificar aquelas dificuldades mais acentuadas que, em alguns casos, podem nem conseguir manipular ou copiar desenhos complexos como o cubo e o relógio.

Um estudo de múltiplos casos que utiliza o quadrado refere poucas, ou nenhuma, diferença em indivíduos com comprometimento neurológico (Rodrigues et al., 2011). Já o uso do desenho da flor na avaliação neuropsicológica auxilia na detecção de heminegligência espacial graças à possibilidade do paciente em colocar mais/menos pétalas do lado esquerdo ou direito (Kumral et al., 2012).
O cubo parece estar altamente relacionado à capacidade do indivíduo de compreensão de modelos espaciais e tridimensionais (Gaestel et al., 2006). A Bateria Montreal Cognitive Assessment (MoCA) parece ter uma alta capacidade discriminante de quadros demenciais ao avaliar habilidades visuoespaciais por meio do desenho do cubo e do relógio (Karunaratne, Hanwella, \& Silva, 2011; Rossetti, Lacritz, Cullum, \& Weiner, 2011). Assim como Rodrigues et al. (2011) sugerem, tanto o desenho do relógio quanto o cubo parece diferenciar pacientes com lesões corticais e subcorticais de hemisfério direito.

Os usos da PC como uma forma de avaliação e identificação de possíveis dificuldades relacionadas ao envelhecimento veem sendo muito associado a quadros neurodegenerativos (Aprahamian et a., 2011), justamente porque há um rebaixamento das habilidades visuoespaciais com o decorrer da idade. No entanto, o impacto que a idade pode ter sobre as PC parece ser muito mais explorado numa perspectiva do envelhecimento do que em indivíduos mais jovens. Prejuízos nas PC em indivíduos mais jovens podem não estar associados apenas a um declínio nas habilidades visuoespaciais, e sim ao funcionamento executivo (Shulman, 2000) de componentes como planejamento e organização perceptual (Bozikas et al., 2003). As PC são uma ferramenta eficaz para avaliação das habilidades visuoespaciais e de funcionamento executivo em indivíduos de baixa escolaridade ou analfabetos, justamente porque elas não dependem de um conhecimento de leitura e escrita (Javadi, Zendehbad, Darabi, Khosravifar, \& Noroozian, 2015). Contudo, o uso dessas tarefas em indivíduos com baixa escolaridade deve ser usado com cuidado devido a dificuldades tridimencionais ou de desenhos espaciais elaborados, como o cubo e o relógio, que dependem de um conhecimento anterior prévio.

Sabe-se que o teste do desenho do relógio é altamente utilizado como um instrumento de rastreio cognitivo de quadros neurológicos e demenciais (Aprahamian et a., 2011). Contudo, não há um consenso na forma de aplicação e pontuação que é mais sensível e específica já que muitos autores se baseiam em padrões internacionais (Aprahamian, Martinelli, Neri, \& Yassuda, 2010). Segundo Hamdan \& Hamdan (2009), o desenho do relógio não é sensível por si só para identificar quadros iniciais da doença de Alzheimer. Entretanto, ele tem correlação moderada com o Miniexame do Estado Mental, o instrumento mais utilizado para rastreio cognitivo (Montiel, Cecato, Bartholomeu, \& Martinelli, 2014). Tarefas de PC atingiram uma posição bastante importante no exame neurocognitivo de idades avançadas devido a sua sensibilidade de identificar sinais sugestivos de quadros de neurodegeneração ou de lesões cerebrais (Cejudo-Bolívar, Torrebalda-Fernandez, GuardiaOlmos, \& Peña-Casanova, 1998). 
Tarefas de PC, além de avaliar componentes de habilidades visuoespaciais e visuoconstrutivas, também podem servir para a avaliação das funções executivas (Aprahamian et al., 2011; Royall, Chiodo, \& Polk, 2005) e da atenção (Karunaratne et al., 2011). Tarefas de habilidades visuoconstrutivas também podem ser maneiras de verificar a flexibilidade cognitiva (Adi-Japha, BerberichArtzi, \& Libnawi, 2010), o planejamento (Rey, 1941; Osterrieth, 1944) e a organização (Paula, Costa, Andrade, Ávila, \& Malloy-Diniz, 2016). Percebe-se que essas tarefas são sensíveis no processo de envelhecimento, pois permitem fazer um rastreio da cognição global (Gaestel, et al., 2006). Sabe-se que, no envelhecimento normal, há um declínio no desempenho do componente visuoespacial (Joanette, Belleville, Gely-Nargeot, Ska, \& Valdois 2008), observado em avaliações complexas por meio do teste do relógio (Drag \& Bieliauskas, 2010).

Assim, apesar de o desempenho em tarefas visuoconstrutivas sofrer menor influência da linguagem, podendo ser usadas em quadros demenciais em analfabetos ou pessoas de baixa escolaridade (Javadiet al., 2015), os desenhos considerados mais complexos (cubo e relógio) exigem mais das habilidades associadas à escolarização (Rossetti, et al., 2011). Essa influência é menos esperada em desenhos mais simples, como a margarida/ flor e o quadrado, que parecem sofrer mais influência de componentes atencionais (Kumral et al., 2012; Rodrigues et al., 2011). Portanto, o presente estudo visa, primeiramente, averiguar efeitos principais e interações de idade e de escolaridade em cada item da tarefa de PC do Instrumento de Avaliação Neuropsicológica Breve - NEUPSILIN. Secundariamente, objetiva-se apresentar normas de referência por desempenho para adultos e idosos em cada item do subteste de PC do NEUPSILIN, já que a normas publicadas desse instrumento apenas geram um escore total único a partir da soma de subescores de desempenho nos quatro desenhos diferentes (quadrado, margarida, cubo e relógio).

\section{Método}

\section{Participantes}

De uma amostra de 627 participantes, inicialmente, foram excluídos 14 indivíduos, dez por não conseguirem relatar com clareza a quantidade de anos completos de escolaridade e quatro por obterem a pontuação inferior ao ponto de corte sugestivo de sinais demenciais no Miniexame do Estado Mental (Kochhann, Varela, Lisboa, \& Chaves, 2010). Os critérios de inclusão foram (1) idade superior a 19 anos, (2) idade mínima de um ano de escolaridade; (3) falantes do português brasileiro, naturais do Brasil; (4) sem histórico atual ou prévio de doenças neurológicas e psiquiátricas autorrelatadas; (5) sem história de uso ou abuso atual ou prévio de drogas. Já os critérios de exclusão (1) apresentar o MEEM inferior ao ponto de corte sugerido por Kochhann, Varela, Lisboa e Chaves, 2010; (2) obter escore acima de seis pontos na Escala de Depressão Geriátrica (GDS-15), o que sugere sintomas depressivos. Esses critérios foram mantidos conforme a bateria do NEUPSILIN original (Fonseca, Salles, \& Parente, 2009).

Assim, participaram desta pesquisa, 613 participantes que foram divididos em quatro grupos etários: jovens de 19 a 39 anos $(n=226)$, adultos intermediários de 40 a 59 anos $(n=146)$, adultos idosos de 60 a 75 anos $(n=140)$, longevos de 76 a 90 anos $(n=101)$. Já a escolaridade foi divida em baixa (um a quatro anos de estudo), intermediária (cinco a oito anos) e alta (nove ou mais anos de escolaridade), mantendo-se assim a distribuição etária e por escolaridade original do NEUPSILIN. A seguir, na Tabela 1, são apresentados os dados sociodemográficos da amostra.

Tabela 1

Dados Sociodemográficos da Amostra

\begin{tabular}{|c|c|c|c|c|c|}
\hline & \multirow[t]{2}{*}{ Variáveis } & $\begin{array}{c}\text { Jovens } \\
\text { 19-39 anos }\end{array}$ & $\begin{array}{c}\text { Intermediária } \\
40-59 \text { anos }\end{array}$ & $\begin{array}{c}\text { Idosos } \\
60-75 \text { anos } \\
\end{array}$ & $\begin{array}{c}\text { Longevos } \\
76-90 \text { anos }\end{array}$ \\
\hline & & $\mathrm{M}(\mathrm{DP})$ & $\mathrm{M}(\mathrm{DP})$ & $\mathrm{M}(\mathrm{DP})$ & $\mathrm{M}(\mathrm{DP})$ \\
\hline \multirow[t]{2}{*}{ Idade } & & $27,07(6,53)$ & $49,36(5.47)$ & $67,06(4,93)$ & $80,92(4,10)$ \\
\hline & 1 a 4 anos & $3,48(0,76)$ & $3,19(1,01)$ & $3,28(0,88)$ & $3,03(1,05)$ \\
\hline \multirow[t]{3}{*}{ Escolaridade } & 5 a 8 anos & $6,75(1,05)$ & $6,58(1,27)$ & $6,00(1,27)$ & $6,33(1,18)$ \\
\hline & 9 ou mais & $13,47(2,72)$ & $15,23(4,73)$ & $13,29(3,09)$ & $13,45(3,53)$ \\
\hline & & $n(\%)$ & $n(\%)$ & $n(\%)$ & $n(\%)$ \\
\hline \multirow{2}{*}{ Gênero } & Feminino & $133(30,40)$ & $113(25,80)$ & $109(25,00)$ & $82(18,80)$ \\
\hline & Masculino & $93(52,80)$ & $33(18,70)$ & $31(17,60)$ & $19(10,80)$ \\
\hline
\end{tabular}

Nota. $\mathrm{M}=$ média, $\mathrm{DP}=$ desvio padrão 


\section{Instrumentos e Procedimentos}

Todos os participantes foram avaliados individualmente em salas claras, silenciosas, ventiladas e livre de estímulos externos. Os instrumentos foram separados entre os instrumentos de caracterização da amostra e a tarefa de avaliação cognitiva.

Questionário de dados socioculturais e aspectos da saúde para avaliar os critérios de inclusão e exclusão e dados de caracterização da amostra (Fonseca et al., 2009).

Miniexame do Estado Mental (Folstein, Folstein, \& Hugh, 1975; Chaves \& Izquierdo, 1992, pontos de corte utilizados Kochhann, Varela, Lisboa, \& Chaves, 2010). É um instrumento de screening para avaliação da cognição global que avalia os domínios de orientação temporal e espacial, memória imediata, atenção e cálculo, evocação recente de palavras e linguagem oral e escrita. Sua pontuação varia de zero a 30 pontos. Os pontos de corte utilizados foram 21 pontos para analfabetos, 22 pontos para escolaridade de um a cinco anos, 23 pontos para escolaridade entre seis e 11 anos e 24 para alta escolaridade (12 anos ou mais).

Escala de Depressão Geriátrica (GDS-15) de Yesavage (Yesavage, Brink, Rose, Lun, Huang, Adey, \& Leirer, 1982, normas utilizadas Almeida \& Almeida, 1999). É uma escala para avaliação de sintomas depressivos que possui 15 perguntas com respostas de sim ou não. Essas perguntas têm como objetivo caracterizar os sintomas de depressão. Sua pontuação varia de 0 a 15 pontos e pode ser classificada como ausência de depressão (0 a 5 pontos), depressão leve a moderada (6 a 10 pontos) e depressão grave (10 pontos ou mais).

Instrumento de Avaliação Neuropsicológica Breve - NEUPSILIN (Fonseca, Salles, \& Parente, 2008, 2009). É uma bateria de múltiplos domínios cognitivos. Utilizou-se o subteste de PC que consiste na cópia de três figuras: o quadrado, a margarida e o cubo; e na solicitação de desenho de um relógio com a soma total, podendo variar de 0 a 16 pontos. Na cópia do quadrado, o indivíduo deve fazer uma forma semelhante a um quadrado mesmo se os vértices não estejam totalmente fechados e os ângulos totalmente retos; a pontuação varia de zero a três pontos. Na cópia da margarida, o desenho deve conter o mesmo número de pétalas (8), ter caule, miolo e sem alterações importantes de proporção e forma. A pontuação da margarida varia de zero a três pontos. A cópia do item do cubo, sua pontuação pode ser de zero a cinco pontos no qual é avaliada a perspectiva de três planos e os vértices totalmente fechados sem erros. Já no item do relógio, solicita-se que o participante desenhe um relógio com os ponteiros marcando $15 \mathrm{~h} 45 \mathrm{~min}$, sua pontuação pode ser de zero a cinco pontos e os itens avaliados são assimetria e quantidade correta dos ponteiros, ponteiro dos minutos localizando o número nove e o ponteiro da hora localizado entre o número três e quatro, disposição espacial dos números e todos os números caracterizados corretamente do zero a doze. Os coeficientes de teste-reteste dos escores total de praxia construtiva foram de 0,943 (Fonseca et al., 2009).

\section{Análise de Dados e Aspectos Éticos}

O estudo foi aprovado pelo Comitê de Ética em Pesquisa da Universidade Federal do Rio Grande do Sul, sob o protocolo número 2006530. Todos os participantes assinaram o Termo de Consentimento Livre e Esclarecido (TCLE). As análises descritivas e inferenciais foram feitas pelo IBM SPSS Statistics versão 20. Realizouse uma análise descritiva de frequências, média e desvio padrão para caracterização das variáveis sociodemográficas. Uma análise de qui-quadrado para a comparação da distribuição de gênero.

Foi realizada uma análise de oneway Anova para comparar o desempenho de cada item do subteste do NEUPSILIN PC nos diferentes grupos, considerando baixa (1 a 4 anos de estudo), intermediária (5 a 8 anos) e alta escolaridade (9 ou mais) e, nos diferentes grupos etários de jovens (19 a 39 anos), adultos intermediários (40 a 59 anos), adultos idosos (60 a 75 anos) e longevos (76 a 90 anos), seguida de análise de post hoc Bonferroni para a comparação do desempenho entre os grupos. Foi realizada uma análise Anova multivariada covariando para idade e escolaridade e possíveis interações dessas variáveis em cada item do subteste do NEUPSLIN.

\section{Resultados}

Na Tabela 2, estão apresentados os resultados obtidos por meio da análise MANCOVA para as PC em que mostra a interação de idade e escolaridade nos itens de cubo e margarida. O tamanho de efeito dessas interações de idade e escolaridade para o item do cubo e para a margarida foi classificado como fraco. Além disso, houve efeito principal de escolaridade no item do quadrado e do relógio e, também, teve efeito principal de idade no relógio. Em relação ao tamanho de efeito, do efeito de idade, o relógio teve uma significância fraca, entretanto, em relação ao efeito com a escolaridade o relógio obteve uma significância média.

$\mathrm{Na}$ Tabela 3, pode-se identificar os resultados de análises comparativas entre os grupos etários e educacionais, os itens das PC que tiveram efeito principal de idade ou de escolaridade.

De maneira geral, tanto no item da margarida quanto no cubo, há um aumento no desempenho conforme aumenta a escolaridade. Em contrapartida, há uma diminuição no desempenho com o aumento da idade. Os grupos que apresentaram diferenças significativas na análise de post hoc Bonferroni podem ser vistos na Tabela 4. Os demais não tiveram diferenças significativas. 
Tabela 2

Efeito de Idade, Escolaridade e Interação nas Praxias Construtivas

\begin{tabular}{|c|c|c|c|c|c|c|c|}
\hline & \multirow[t]{2}{*}{ Itens PC } & $\begin{array}{l}\text { Soma dos } \\
\text { quadrados }\end{array}$ & gl & $\begin{array}{c}\text { Quadrados } \\
\text { médios }\end{array}$ & $\mathrm{F}$ & Sig. & Partial eta \\
\hline & & \multicolumn{6}{|c|}{ Idade } \\
\hline Quadrado & & 0,29 & 3 & 0,098 & 0,74 & 0,526 & 0,004 \\
\hline Margarida & & 3,33 & 3 & 1,109 & 4,46 & 0,004 & 0,021 \\
\hline Cubo & & 7,74 & 3 & 2,579 & 1,62 & 0,183 & 0,008 \\
\hline Relógio & & 54,48 & 3 & 18,162 & 9,58 & $<0,001$ & 0,045 \\
\hline & \multirow[t]{2}{*}{ Itens PC } & $\begin{array}{l}\text { Soma dos } \\
\text { quadrados }\end{array}$ & $g l$ & $\begin{array}{l}\text { Quadrados } \\
\text { médios }\end{array}$ & $\mathrm{F}$ & Sig. & $\begin{array}{c}\text { Partial } \\
\text { eta }\end{array}$ \\
\hline & & \multicolumn{6}{|c|}{ Escolaridade } \\
\hline Quadrado & & 1,52 & 2 & 0,763 & 5,77 & 0,003 & 0,018 \\
\hline Margarida & & 11,28 & 2 & 5,644 & 22,72 & $<0,001$ & 0,069 \\
\hline Cubo & & 321,78 & 2 & 160,89 & 101,21 & $<0,001$ & 0,248 \\
\hline Relógio & & 145,94 & 2 & 72,970 & 38,50 & $<0,001$ & 0,111 \\
\hline & \multirow[t]{2}{*}{ Itens PC } & $\begin{array}{l}\text { Soma dos } \\
\text { quadrados }\end{array}$ & gl & $\begin{array}{c}\text { Quadrados } \\
\text { médos }\end{array}$ & $\mathrm{F}$ & Sig. & Partial eta \\
\hline & & \multicolumn{6}{|c|}{ Idade-Escolaridade } \\
\hline Quadrado & & 1,017 & 6 & 0,169 & 1,28 & 0,263 & 0,012 \\
\hline Margarida & & 4,161 & 6 & 0,694 & 2,79 & 0,011 & 0,027 \\
\hline Cubo & & 20,494 & 6 & 3,146 & 2,15 & 0,046 & 0,021 \\
\hline Relógio & & 9,603 & 6 & 1,601 & 0,84 & 0,536 & 0,008 \\
\hline
\end{tabular}

Nota. Sig. $\leq 0,05$ significante

Tabela 3

Comparação entre Idade e Escolaridade nos Grupos Etários e de Escolaridade

\begin{tabular}{|c|c|c|c|c|c|c|}
\hline Efeito principal & Interação entre os grupos* & $\begin{array}{c}\text { Diferença } \\
\text { média }\end{array}$ & $\begin{array}{c}\text { Erro } \\
\text { padrão }\end{array}$ & Sig. & $\begin{array}{l}\text { Limite } \\
\text { inferior }\end{array}$ & $\begin{array}{c}\text { Limite } \\
\text { superior }\end{array}$ \\
\hline \multicolumn{7}{|c|}{ Idade } \\
\hline \multirow{6}{*}{ Relógio } & $\mathrm{AJ} \mathrm{X} \mathrm{AI}$ & 0,072 & 0,151 & 1,000 & $-0,327$ & 0,471 \\
\hline & AJ X AId & 0,441 & 0,150 & 0,021 & 0,043 & 0,839 \\
\hline & AJ X LO & 0,821 & 0,169 & $<0,001$ & 0,374 & 1,268 \\
\hline & AI X AId & 0,369 & 0,163 & 0,144 & $-0,063$ & 0,802 \\
\hline & AI X LO & 0,749 & 0,180 & $<0,001$ & 0,271 & 1,226 \\
\hline & AId X LO & 0,380 & 0,180 & 0,213 & $-0,097$ & 0,856 \\
\hline \multicolumn{7}{|c|}{ Escolaridade } \\
\hline \multirow{3}{*}{ Quadrado } & 1-4 idade X 5-8 idade & $-0,031$ & 0,040 & 1,000 & $-0,127$ & 0,065 \\
\hline & 1-4 idade X 9 ou mais & $-0,119^{*}$ & 0,038 & 0,005 & $-0,209$ & $-0,029$ \\
\hline & 5-8 idade X 9 ou mais & $-0,088^{*}$ & 0,036 & 0,047 & $-0,175$ & $-0,001$ \\
\hline \multirow{3}{*}{ Relógio } & 1-4 idade X 5-8 idade & $-0,743^{*}$ & 0,152 & $<0,001$ & $-1,108$ & $-0,378$ \\
\hline & 1-4 idade X 9 ou mais & $-1,247^{*}$ & 0,142 & $<0,001$ & $-1,589$ & $-0,906$ \\
\hline & 5-8 idade X 9 ou mais & $-0,505^{*}$ & 0,137 & 0,001 & $-0,833$ & $-0,176$ \\
\hline
\end{tabular}

Nota. Sig. $\leq 0.05$ significante; AJ=adulto jovem, AI=adulto intermediário, AId=adulto idoso, LO=adulto longevo

$\mathrm{Na}$ Tabela 5, pode-se visualizar a média e o desvio padrão de cada grupo, de acordo com a faixa etária e a escolaridade em cada item do subteste de PC.

Sugere-se a análise de ocorrência de déficits por meio do cálculo do escore Z (escore do participante
- média do grupo $\div$ desvio padrão) (Lezak, 2012; Fonseca et al., 2009) com pontos de corte, considerando-se grupos educacionais para o quadrado e grupos mistos por idade versus escolaridade para margarida, cubo e relógio. 
Tabela 4

Post Hoc Bonferroni nos Grupos versus Educação

\begin{tabular}{|c|c|c|c|c|c|c|}
\hline Itens PC & Interação entre os grupos & $\begin{array}{c}\text { Diferença } \\
\text { média }\end{array}$ & $\begin{array}{c}\text { Erro } \\
\text { padrão }\end{array}$ & Sig. & $\begin{array}{l}\text { Limite } \\
\text { inferior }\end{array}$ & $\begin{array}{c}\text { Limite } \\
\text { superior }\end{array}$ \\
\hline \multirow{15}{*}{ Margarida } & AJ baixa X AI alta & $-0,45^{*}$ & 0,083 & $<0,001$ & $-0,73$ & $-0,17$ \\
\hline & AJ baixa X AI intermediária & $-0,34^{*}$ & 0,100 & 0,049 & $-0,68$ & 0,00 \\
\hline & AJ intermediária X AJ alta & $-0,41^{*}$ & 0,079 & $<0,001$ & $-0,68$ & $-0,15$ \\
\hline & AJ alta X AI baixa & $0,55^{*}$ & 0,089 & $<0,001$ & 0,25 & 0,85 \\
\hline & AJ alta X AId baixa & $0,47^{*}$ & 0,091 & $<0,001$ & 0,16 & 0,78 \\
\hline & AJ alta X AId intermediária & $0,39^{*}$ & 0,083 & $<0,001$ & 0,11 & 0,67 \\
\hline & AJ alta X LO baixa & $0,47^{*}$ & 0,091 & $<0,001$ & 0,16 & 0,78 \\
\hline & AJ alta X LO intermediária & $0,52^{*}$ & 0,101 & $<0,001$ & 0,17 & 0,86 \\
\hline & AJ alta X LO alta & $0,32^{*}$ & 0,088 & 0,024 & 0,02 & 0,61 \\
\hline & AI baixa X AI intermediária & $-0,44^{*}$ & 0,105 & 0,002 & $-0,79$ & $-0,08$ \\
\hline & AI baixa X AI alta & $-0,42^{*}$ & 0,101 & 0,002 & $-0,76$ & $-0,08$ \\
\hline & AI intermediária X LO baixa & $0,47^{*}$ & 0,117 & 0,004 & 0,08 & 0,87 \\
\hline & AI intermediária X LO intermediária & $0,40^{*}$ & 0,116 & 0,035 & 0,01 & 0,80 \\
\hline & AI alta X LO baixa & $0,46^{*}$ & 0,114 & 0,005 & 0,07 & 0,84 \\
\hline & AI alta X LO intermediária & $0,39^{*}$ & 0,112 & 0,040 & 0,01 & 0,77 \\
\hline \multirow{35}{*}{ Cubo } & AJ baixa X AJ intermediária & $-0,93^{*}$ & 0,241 & 0,008 & $-1,75$ & $-0,11$ \\
\hline & AJ baixa X AJ alta & $-2,12^{*}$ & 0,210 & $<0,001$ & $-2,83$ & $-1,41$ \\
\hline & AJ baixa X AI intermediária & $-1,05^{*}$ & 0,254 & 0,003 & $-1,91$ & $-0,19$ \\
\hline & AJ baixa X AI alta & $-1,92^{*}$ & 0,243 & $<0,001$ & $-2,74$ & $-1,09$ \\
\hline & AJ baixa X AId alta & $-1,68^{*}$ & 0,245 & $<0,001$ & $-2,51$ & $-0,85$ \\
\hline & AJ baixa X LO alta & $-1,89^{*}$ & 0,261 & $<0,001$ & $-2,77$ & $-1,00$ \\
\hline & AJ intermediária X AJ alta & $-1,19^{*}$ & 0,199 & $<0,001$ & $-1,87$ & $-0,52$ \\
\hline & AJ intermediária X AI alta & $-0,98^{*}$ & 0,234 & 0,002 & $-1,78$ & $-0,19$ \\
\hline & AJ intermediária X LO baixa & $1,24^{*}$ & 0,286 & 0,001 & 0,27 & 2,21 \\
\hline & AJ intermediária X LO alta & $-0,96^{*}$ & 0,253 & 0,011 & $-1,81$ & $-0,10$ \\
\hline & AJ alta X AI baixa & $2,04^{*}$ & 0,225 & $<0,001$ & 1,28 & 2,81 \\
\hline & AJ alta X AI intermediária & $1,07^{*}$ & 0,214 & $<0,001$ & 0,35 & 1,80 \\
\hline & AJ alta X AId baixa & $1,51^{*}$ & 0,231 & $<0,001$ & 0,73 & 2,30 \\
\hline & AJ alta X AId intermediária & $1,34^{*}$ & 0,211 & $<0,001$ & 0,62 & 2,05 \\
\hline & AJ alta X LO baixa & $2,43^{*}$ & 0,260 & $<0,001$ & 1,55 & 3,31 \\
\hline & AJ alta X LO intermediária & $1,56^{*}$ & 0,257 & $<0,001$ & 0,69 & 2,43 \\
\hline & AI baixa X AI intermediária & $-0,97^{*}$ & 0,266 & 0,019 & $-1,87$ & $-0,07$ \\
\hline & AI baixa X AI alta & $-1,84^{*}$ & 0,256 & $<0,001$ & $-2,71$ & $-0,97$ \\
\hline & AI baixa X AId alta & $-1,60^{*}$ & 0,258 & $<0,001$ & $-2,48$ & $-0,73$ \\
\hline & AI baixa X LO alta & $-1,81^{*}$ & 0,274 & $<0,001$ & $-2,73$ & $-0,88$ \\
\hline & AI intermediária X AI alta & $-0,87^{*}$ & 0,247 & 0,033 & $-1,70$ & $-0,03$ \\
\hline & AI intermediária X LO baixa & $1,36^{*}$ & 0,297 & $<0,001$ & 0,36 & 2,36 \\
\hline & AI alta X AId baixa & $1,31^{*}$ & 0,262 & $<0,001$ & 0,42 & 2,20 \\
\hline & AI alta X AId intermediária & $1,13^{*}$ & 0,244 & $<0,001$ & 0,31 & 1,96 \\
\hline & AI alta X LO baixa & $2,23^{*}$ & 0,288 & $<0,001$ & 1,25 & 3,20 \\
\hline & AI alta X LO intermediária & $1,35^{*}$ & 0,284 & $<0,001$ & 0,39 & 2,32 \\
\hline & AId baixa X AId alta & $-1,07^{*}$ & 0,264 & 0,003 & $-1,97$ & $-0,18$ \\
\hline & AId baixa X LO alta & $-1,28^{*}$ & 0,279 & $<0,001$ & $-2,22$ & $-0,34$ \\
\hline & AId intermediária X AId alta & $-0,90^{*}$ & 0,246 & 0,019 & $-1,73$ & $-0,06$ \\
\hline & AId intermediária X LO baixa & $1,09^{*}$ & 0,294 & 0,015 & 0,10 & 2,09 \\
\hline & AId intermediária X LO alta & $-1,10^{*}$ & 0,262 & 0,002 & $-1,99$ & $-0,22$ \\
\hline & AId alta X LO baixa & $1,99^{*}$ & 0,289 & $<0,001$ & 1,01 & 2,97 \\
\hline & AId alta X LO intermediária & $1,12^{*}$ & 0,286 & 0,007 & 0,15 & 2,09 \\
\hline & LO baixa X LO alta & $-2,20^{*}$ & 0,303 & $<0,001$ & $-3,22$ & $-1,17$ \\
\hline & LO intermediária X LO alta & $-1,32^{*}$ & 0,300 & 0,001 & $-2,34$ & $-0,31$ \\
\hline
\end{tabular}

Nota. Sig. $\leq 0.05$ significante; AJ=adulto jovem, AI=adulto intermediário, AId=adulto idoso, LO=adulto longevo; baixa (1-4 idade), intermediária (5-8 idade) e alta (9 ou mais) escolaridade 
Tabela 5

Média e Desvio-padrão das PC de Acordo com os Grupos Etários e a Escolaridade para Normas

\begin{tabular}{|c|c|c|c|c|c|c|c|}
\hline \multirow{3}{*}{$\begin{array}{c}\text { Itens } \\
\text { praxias }\end{array}$} & \multirow{3}{*}{ Idade grupo } & \multicolumn{6}{|c|}{ Escolaridade } \\
\hline & & \multicolumn{2}{|c|}{1 - 4 idade } & \multicolumn{2}{|c|}{5 - 8 idade } & \multicolumn{2}{|c|}{9 ou mais } \\
\hline & & M & DP & M & DP & M & $D P$ \\
\hline \multirow{4}{*}{ Quadrado } & 19-39 idade & 2,86 & 0,34 & 2,81 & 0,39 & 2,88 & 0,32 \\
\hline & 40-59 idade & 2,69 & 0,60 & 2,85 & 0,35 & 2,95 & 0,22 \\
\hline & $60-75$ idade & 2,87 & 0,33 & 2,84 & 0,37 & 2,92 & 0,26 \\
\hline & 76-90 idade & 2,76 & 0,43 & 2,80 & 0,41 & 2,93 & 0,26 \\
\hline \multirow{4}{*}{ Margarida } & 19-39 idade & 2,10 & 0,41 & 2,14 & 0,39 & 2,54 & 0,50 \\
\hline & 40-59 idade & 2,00 & 0,49 & 2,44 & 0,50 & 2,41 & 0,53 \\
\hline & $60-75$ idade & 2,08 & 0,42 & 2,16 & 0,51 & 2,35 & 0,55 \\
\hline & 76-90 idade & 1,97 & 0,49 & 2,03 & 0,49 & 2,24 & 0,62 \\
\hline \multirow{4}{*}{ Cubo } & 19-39 idade & 2,14 & 1,34 & 3,07 & 1,32 & 4,22 & 1,02 \\
\hline & 40-59 idade & 2,21 & 1,37 & 3,19 & 1,39 & 4,07 & 1,01 \\
\hline & $60-75$ idade & 2,74 & 1,58 & 2,90 & 1,43 & 3,75 & 1,39 \\
\hline & 76-90 idade & 1,83 & 1,22 & 2,70 & 1,31 & 4,00 & 1,03 \\
\hline \multirow{4}{*}{ Relógio } & 19-39 idade & 2,55 & 1,22 & 2,93 & 1,37 & 3,80 & 1,16 \\
\hline & 40-59 idade & 2,21 & 1,66 & 3,10 & 1,37 & 3,70 & 1,22 \\
\hline & $60-75$ idade & 2,05 & 1,29 & 2,73 & 1,61 & 3,21 & 1,51 \\
\hline & 76-90 idade & 1,55 & 1,40 & 2,60 & 1,54 & 2,71 & 1,36 \\
\hline
\end{tabular}

Nota. M=média, DP=desvio-padrão

\section{Discussão}

O presente estudo teve como objetivo verificar a interação e efeito de idade e escolaridade nas PC para os quatro itens das PC do NEUPSILIN, como também, consequentemente, obter normas de performance por desempenho. Os resultados sugerem que todos os itens das praxias apresentam diferenças significativas entre grupos etários e de escolaridade. Houve interação entre idade e escolaridade nos itens cubo e margarida em que, conforme o aumento da idade menor é o desempenho; já com o aumento da escolaridade melhor o desempenho. O item do quadrado sofreu maior efeito da escolaridade; enquanto o relógio teve efeito tanto de idade, quanto de escolaridade. De forma geral, os grupos de maiores escolaridades tiveram melhores desempenhos quando comparados aos grupos de baixa escolaridade. Adicionalmente, indivíduos mais jovens tiveram melhor desempenho em todos os itens das praxias em comparação aos mais velhos.

$\mathrm{Na}$ medida em que, a partir dos achados descritivos de média e desvio-padão, é possível se calcular o escore $\mathrm{Z}$ e se interpretar presença, ou ausência de déficit. Dessa forma, sugere-se para os itens das praxias construtivas a utilização do ponto de corte $Z-1$ ao invés de $-1,5$ para considerar prejuízo, pois ele parece rastrear melhor as possíveis deficiências encontradas em pacientes com comprometimento cognitivo Leve (Jak et al., 2009; Liepelt-Scarfone et al., 2011). Adicionalmente, pode cobrir uma gama maior de possíveis prejuízos e dificuldades cognitivas (Jak et al., 2009). Para esse cálculo, sugere-se subtrair a pontuação obtida na escala pelo desempenho no grupo normativo ao qual pertence e dividir esse valor pelo desvio padrão do grupo (Lezak, Howieson, Bigler, \& Tranel, 2012).

O quadrado parece não ser um bom item para detecção de prejuízos ao longo do desenvolvimento; entretanto, mostra-se sensível à escolaridade formal. Hipotetiza-se que desenhos como o quadrado são muito simples e com pouca complexidade para ser realizado, mesmo em pacientes com lesão cerebrovascular (Rodrigues et al., 2008). Acredita-se que tal facilidade ocorra por estar ligado a um formato simples e que depende de um modelo representacional aprendido sem dificuldade e, apenas no caso de muita severidade na habilidade construtiva, mostrar-se-á prejudicado. Mesmo com maior nível de facilidade e tendência a efeito de teto, para pacientes muito graves com habilidades visuoconstrutivas mais restritas, pode ser um estímulo útil para rastreio cognitivo.

Em contrapartida, o item da margarida/flor parece ser um bom desenho para discriminar prejuízos relacionados a quadros neurológicos devido à avaliação, como número de pétalas e simetria (Kumral et al., 2012). Isso parece estar relacionado porque o desenho da margarida/ flor serve para detecção de déficits atencionais importantes, como heminegligência visual, distorções espaciais na percepção comuns em pacientes com lesões cerebrais, 
tumores (Kumral et al., 2012), como também déficits sutis na atenção que já são importantes preditores de declínio/prejuízo cognitivo (Farias et al., 2006). Dessa forma, a margarida/flor parece sofrer forte influência da idade devido ao fato de que quanto maior a idade maior a possibilidade de distorções relacionadas à simetria e comportamentos desorganizados (Kumral et al., 2012) devido a um rebaixamento da atenção cognição basal, mesmo em indivíduos adultos idosos saudáveis (Johnson, 2009), o que não é comum em indivíduos mais jovens.

O desenho do cubo parece ter forte influência da escolaridade e na detecção de prejuízos cerebrais importantes. Segundo Gaestel et al (2006), o cubo é uma importante medida para discriminar prejuízos em pacientes com doença de Alzheimer, quando comparados ao envelhecimento normal. No cubo, por este ser uma figura tridimensional, indivíduos analfabetos ou com baixa escolaridade apresentam muita dificuldade, podendo simular déficits, não por causa de apraxias, e sim pela baixa escolaridade (Robbins et al., 2013). Assim alguns itens, como o cubo, podem depender mais da escolaridade e, por isso, deve-se considerar as normas de acordo com os anos de educação formal.

Diferentemente do cubo, o relógio não parece depender de conhecimentos tridimensionais, mas de uma representação mental, principalmente por não depender de modelo externo a da habilidade de cópia, o que o torna mais suscetível à identificação de prejuízos decorrentes do declínio cognitivo (Price et al., 2011). Os resultados encontrados indicaram que o relógio teve efeito apenas da idade, demonstrando que indivíduos mais velhos tinham mais dificuldade no desenho. Assim, percebe-se que o relógio é uma ótima ferramenta de avaliação ecológica e visuoperceptual (Santana et a., 2013) muito utilizada como rastreio e triagem de pacientes com demência (Price et al., 2011). É a partir do item do relógio que se pode rastrear vários componentes do funcionamento executivo (Shulman, 2000), como flexibilidade cognitiva (Adi-Japha et al., 2010), organização e planejamento espacial (Bozikas et al., 2003), memória de trabalho (para recordar da instrução verbal e, ao mesmo tempo, realizar o desenho) (Price et al., 2011).

As funções executivas para Diamond (2013) são essencialmente importantes, servindo como base para habilidades cognitivas emocionais, sociais e aspectos de vida. Prejuízos nesses componentes são comumente vistos no declínio cognitivo devido a doenças neurológicas (Price et al., 2011; Montiel et al., 2014) ou psiquiátricas (Cotrena et al., 2016). Somente o item do relógio, na Bateria NEUPSILIN, exige de uma representação mental pré-existente, diferente dos demais itens, que é apenas a cópia do desenho.

Apesar dos tamanhos de efeito da interação entre idade e escolaridade, tanto para a margarida quanto para o cubo, terem sido fracas, percebe-se que figuras de PC mais simples como essas sofrem influência de ambos os fatores socioculturais, ainda que tarefas de PC sejam relevantes, pois não dependem da leitura e da escrita, o que dificultaria a avaliação de indivíduos com baixa escolaridade ou iletrados (Javadi et al., 2015). Mesmo que a cópia do quadrado sofra baixa influência da idade e que este seja um desenho simples para pacientes com transtornos neurológicos graves ou com quadros degenerativos, ele pode ser útil como um rastreio para se avaliar o desempenho em idosos mais longevos. $\mathrm{O}$ desenho do relógio tem o mesmo tipo de tamanho de efeito em relação à idade, mas demonstra um tamanho de efeito médio com relação à escolaridade. Comparado aos outros desenhos de PC, até os geométricos, como o cubo, o relógio se mostra mais sensível ao efeito da escolaridade. Esse efeito da escolaridade no relógio demonstra a importância da representação mental, do efeito de componentes executivos de planejamento e de organização perceptual que o indivíduo necessita para sua execução (Bozikas et al., 2003; Shulman, 2000).

Com isso a educação foi a variável que mostrou efeito desde o item mais simples até o mais complexo nas PC. Esse aspecto demonstra a importância de se considerar o nível de escolaridade nas normas de todos os instrumentos, sejam eles verbais ou não verbais (Javadi et al., 2015). Além de que elas também auxiliam na verificação de prejuízos visuoespaciais e na detecção de dificuldades em outros componentes cognitivos que possam estar comprometidos, como atenção, funções executivas e habilidades motoras (Pena et al., 2008).

As PC, além de serem atividades de rápida aplicabilidade, podem ser usadas como rastreio em entrevistas ou em avaliações breves da cognição. No entanto, uma das limitações encontradas neste estudo é que elas funcionam muito bem para a detecção de prejuízos importantes, mas limitam-se na identificação de prejuízos sutis, ou alerta de déficits que podem passar despercebidos, ou sem alterações em tarefas dessa modalidade. Tal limitação está ligada também a outro limite inerente deste estudo em que as tarefas de PC da bateria NEUPSILIN têm poucos itens com escores e subescores com faixa reduzida de variabilidade (variando 0,1 ou 2 pontos em alguns casos como o desenho do quadrado). Dessa forma, recomenda-se a realização dessas tarefas apenas como primeiro passo de exame de habilidades visuoespaciais e construtivas, assim como de indícios de algumas dificuldades executivas ligadas às praxias, devendo ser complementadas por avaliações mais expandidas.

A avaliação de PC auxilia, principalmente, na detecção de prejuízos no envelhecimento (Handam \& Handam, 2009). No entanto, ela se mostra sensível para adultos jovens e intermediários, servindo como uma ferramenta eficaz para a prática clínica e hospitalar a sua aplicabilidade. Espera-se que tais dados normativos sejam úteis no exame de diagnóstico diferencial de ocorrência de déficits por quadros clínicos ao invés de 
falsos positivos por menor escolarização ou em idade mais avançada. Futuramente deve-se refletir sobre possíveis pontos de corte para as PC em quadros demenciais, como também sua comparação com outras medidas cognitivas para ver se há relação, ou não com componentes atencionais, executivos, mnemônicos e de linguagem.

\section{Referências}

Adi-Japha, E., Berberich-Artzi, J., \& Libnawi, A. (2010). Cognitive flexibility in drawings of bilingual children. Child Development, 81(5), 1356-1366. doi: 10.1111/j.1467-8624.2010.01477.x

Almeida, O. P., \& Almeida, S. A. (1999). Confiabilidade da versão brasileira da escala de depressão em geriatria (GDS) versão reduzida. Arquivos de Neuro-psiquiatria, 57(2-B), 421-426. doi:10.1590/S0004-282X1999000300013

Aprahamian, I., Martinelli, J. E., Cecato, J., Izbicki, R., \& Yassuda, M. S. (2011). Can the CAMCOG be a good cognitive test for patients with Alzheimer's disease with low levels of education? International psychogeriatrics, 23(1), 96-101. doi: 10.1017/S104161021000116X

Aprahamian. I.. Martinelli. J. E.. Neri. A. L.. \& Yassuda. M. S. (2010). The accuracy of the Clock Drawing Test compared to that of standard screening tests for Alzheimer's disease: Results from a study of Brazilian elderly with heterogeneous educational backgrounds. International psychogeriatrics, 22(1). 64-71. doi: 10.1017/S1041610209991141

Bozikas, V. P., Kosmidis, M. H., Kourtis, A., Gamvrula, K., Melissidis, P., Tsolaki, M., \& Karavatos, A. (2003). Clock drawing test in institutionalized patients with schizophrenia compared with Alzheimer's disease patients. Schizophrenia research, 59(2-3), 173-179. doi: 10.1016/S0920-9964(01)00335-8

Cejudo-Bolívar, J. C., Torrealba-Fernández, E., Guardia-Olmos, J., \& Peña-Casanova, J. (1998). Praxis constructiva a la copia: Normas ampliadas del test Barcelona. Neurología, 13(7), 329-334. Recuperado de https://docplayer.es/18858852-Praxis-constructiva-a-la-copianormas-ampliadas-del-test-barcelona.html

Chaves, M. L. F., \& Izquierdo, I. A. (1992). Differential diagnosis between dementia and depression: a study of efficiency increment. Acta Neurology Scandinava, 85, 378-382. doi:10.1111/j.1600-0404.1992.tb06032.x

Cotrena, C., Branco, L. D., Shansis, F. M., \& Fonseca, R. P. (2016). Executive function impairments in depression and bipolar disorder: Association with functional impairment and quality of life. Journal of Affective Disorders, 5(190), 744-753. doi: 10.1016/j.jad.2015.11.007

Diamond, A. (2013). Executive functions. Annual Review of psychology, 64, 135-68. doi: 10.1146/annurev-psych-113011-143750

Drag, L. L. \& Bieliauskas, L. A. (2010). Contemporary Review 2009: Cognitive Aging. Journal of Geriatric Psychiatric and Neurology, 23(2), 75-93. doi: 10.1177/0891988709358590

Elderkin-Thompson, V., Kumar, A., Mintz, J., Boone, K., Bahng, E., \& Lavretsky, H. (2004). Executive dysfunction and visuospatial ability among depressed elders in a community setting. Archive of Clinical Neuropsychology, 19(5), 597-611. doi: 10.1016/j.acn.2003.08.009

Farias, S. T., Mungas, D., Reed, B. R., Harvey, D., Cahn-Weiner, D., \& Decarli, C. (2006). MCI is associated with deficits in everyday functioning. Alzheimer Disease and Associated Disorders, 20(4), 217-223. doi: 10.1097/01.wad.0000213849.51495.d9

Fillenbaum, G. G., Heyman, A., Huber, M. S., Ganguli, M. \& Unverzagt, F. W. (2001). Performance of elderly African American and White community residents on the CERAD Neuropsychological Battery. Journal of the international neuropsychological society, 7(4), 502-509.

Fillenbaum, G. G., McCurry, S. M., Kuchibhatla, M., Masaki, K. H., Borenstein, A. R., Foley, D. J., Heyman, A., Larson, E. B., \& White L. (2005). Performance on the CERAD neuropsychology battery of two samples of Japanese-American elders: Norms for persons with and without dementia. Journal of international neuropsychological society, 11(2), 192-201.

Folstein, M. F., Folstein, S. E., \& McHugh, P. R. (1975). "Mini-Mental State": A practical method for grading the cognitive state of patients for the clinician. Journal of Psychiatric Research, 12(3), 189-198. doi: 10.1016/0022-3956(75)90026-6

Fonseca, R. P., Salles, J. F., \& Parente, M. A. M. P. (2008). Development and content validity of the Brazilian Brief Neuropsychological Assessment Battery NEUPSILIN. Psychology \& Neuroscience, 1(1), 55-62. doi: 10.3922/j.psns.2008.1.009

Fonseca, R. P., Salles, J. F. de, \& Parente, M. A. de M. P. (2009). Neupsilin - Instrumento de Avaliação Neuropsicológica Breve. Vetor editora: São Paulo.

Fonseca, R. P., Zimmermann, N., Pawlowski, J., Oliveira, C. R. De, Gindri, G., Scherer, L. C., \& ... Parente, M. A. de M. P. (2012). Métodos em neuropsicologia. In J. Landeira-Fernandez \& S. S. Fukusima (Eds.), Métodos em neurociência. Editora Manole: São Paulo.

Freeman, R. Q., Giovannetti, T., Lamar, M., Cloud, B. S., Stern, R. A., Kaplan, E., \& Libon, D. J. (2000). Visuoconstructional problems in dementia: Contribution of executive systems functions. Neuropsychology, 14(3), 415-426. doi: 10.1037/0894-4105.14.3.415

Gaestel, Y., Amieva, H., Letenneur, L., Dartigues, J. F., \& Fabrigoule, C. (2006). Cube drawing performances in normal ageing and Alzheimer's disease: Data from the PAQUID elderly population-based cohort. Dementia and Geriatric Cognitive Disorders, 21(1), 22-32. doi:10.1159/000089216

Gragnaniello, D., Kessler, J., Bley, M., \& Mielke, R. (1998). Copying and free drawing by patients with Alzheimer disease of different dementia stages. Der Nervenarzt, 69(11), 991-998.

Hamdan, A. C., \& Hamdan, E. M. L. R. (2009). Teste do desenho do relógio: Desempenho de idosos com doença de Alzheimer. Revista Brasileira de Ciências do Envelhecimento Humano, 6(1), 98-105. doi:10.5335/rbceh.2012.364

Hubbard, E. J., Santini, V., Blankevoort, C. G., Volkers, K. M., Barrup, M. S., Byerly, L., Chaisson, C., Jefferson, A. L., Kaplan, E., Green, R. C., \& Stern, R.A. (2008). Clock drawing performance in cognitively normal elderly. Archives of Clinical Neuropsychology, 23(3), $295-327$. doi: 10.1016/j.acn.2007.12.003

Instituto Brasileiro de Geografia e Estatística - IBGE. (2002). Perfil dos Idosos Responsáveis pelos Domicílios. Recuperado de http://www.ibge.gov. br/home/presidencia/noticias/25072002pidoso.shtm

Jak, A. J., Bondi, M. W., Delano-Wood, L., Wierenga, C., Corey-Bloom, J., Salmon, D. P., \& Delis, D. C. (2009). Quantification of five neuropsychological approaches to defining mild cognitive impairment. The American journal of geriatric psychiatric, 17(5), 368-375. doi: 10.1097/JGP.0b013e31819431d5

Javadi, P. S., Zendehbad, A., Darabi, F., Khosravifar, S., \& Noroozian, M. (2015). Development and implementation of persian test of elderly for assessment of cognition and executive function (PEACE). Electronic physician, 7(7), 1549-1556. doi: 10.19082/1549 
Joanette, Y., Belleville, S., Gely-Nargeot, M. C., Ska, B., \& Valdois, S. (2000). Pluralité des patrons d'atteinte cognitive accompagnant le vieillissement normal et la démence. Revue Neurologique 156(8-9), 759-766. doi : RN-09-2000-156-8-9-0035-3787-101019-ART54

Johnson, J. (2009). Mild Cognitive Impairment subgroups. Em Miller BL \& Boeve BF (Eds.), The Behavioral Neurology of Dementia (pp. 172-187). Cambridge: Cambridge University Press.

Karunaratne, S., Hanwella, R., \& Silva, V. de (2011). Validation of the Sinhala version of the Montreal Cognitive Assessment in screening for dementia. Ceylon Medical Journal, 56(4), 147-153. doi: 10.4038/cmj.v56i4.3892

Kochhann, R., Varela, J. S., Lisboa, C. S. de M., \& Chaves, M. L. F. (2010). The Mini Mental State Examination Review of cutoff points adjusted for schooling in a large Southern Brazilian sample. Dementia \& Neuropsychology, 4(1), 35-41. doi:10.1590/S198057642010DN40100006

Kumral, E., EvAJpan, D., Sirin, T. \& Alpaydin, S. (2012). Hyposchematia without spatial neglect in 2 patients with lesions involving the insula. Cognitive and behavioral neurology, 25(3), 149-153. doi: 10.1097/WNN.0b013e31826b70de

Lezak, M. (2012). Neuropsychological Assessment. New York, NY: Oxford University Press.

Lezak, M. D., Howieson, D. B., Bigler, E. D., \& Tranel, D. (2012). Neuropsychological Assessment. (M. D. Lezak, D. B. Howieson, E. D. Bigler, \& D. Tranel, Eds.) (5th ed.). New York: Oxford University Press.

Liepelt-Scarfone, I., Graeber, S., Feseker, A., Baysal, G., Godau, J., Gaenslen, A., Maetzler, W., \& Berg, D. (2011). Influence of different cut-off values on the diagnosis of mild cognitive impairment in Parkinson's disease. Parkinsons Disease, 540842, 1-7. doi: 10.4061/2011/540843

Montiel, J. M., Cecato, J. F., Bartholomeu, D., \& Martinelli, J. E. (2014). Testes do desenho do relógio e de fluência verbal: Contribuição diagnóstica para o Alzheimer. Revista Psicologia: Teoria e Prática, 16(1), 169-180. doi: 10.15348/1980-6906/psicologia.v16n1p169-180

Oliveira, M. S. \& Rigoni, M. S. (2010). Figuras complexas de Rey. Casa do Psicólogo: Porto Alegre.

Osterrieth, P. A. (1944). The test of copying a complex figure: A contribution to the study of perception and memory. Archives de Psychologie, 30, 286-356.

Parsey, C. M., \& Schmitter-Edgecombe, M. (2011). Quantitative and qualitative analyses of the clock drawing test in mild cognitive impairment and Alzheimer disease: Evaluation of a modified scoring system. Journal of geriatric, psychiatric and neurology, 24(2), 108-118. doi: 10.1177/0891988711402349

Paula, J. J. de, Costa, M. V., Andrade, G de F., Ávila R. T., \& Malloy-Diniz, L. (2016). Validity and reliability of a "simplified" version of the Taylor Complex Figure Test for the assessment of older adults with baixa formal education. Dementia \& Neuropsychology, 10(1), 52-57. doi:10.1590/s1980-57642016dn10100010.

Pena, M., Sobreira, E., Souza, C., Oliveira, G., Tumas, V., \& Vale, F. (2008). Visuospatial cognitive tests for the evaluation of patients with Parkinson's disease. Dementia \& Neuropsychologia, 2(3), 201-205. doi: 10.1590/S1980-57642009DN20300007

Price, C. C., Cunningham, H., Coronado, N., Freedland, A., Cosentino, S., Penney, D. L., Penisi, A., Bowers, D., Okun, M. S., \& Libon, D. J. (2011). Clock Drawing in the Montreal Cognitive Assessment: Recommendations for Dementia Assessment. Dementia and geriatric cognitive disorders, 31(3), 179-187. doi: 10.1159/000324639

Rey, A. (1941). L'examen psychologique dans les cas d'encephalopathie traumatique. Archives de Psychologie, 28, 215-285.

Robbins, R. N., Joska, J. A., Thomas, K. G., Stein, D. J., Linda, T., Mellins, C. A., \& Remien, R. H. (2013). Exploring the utility of the Montreal Cognitive Assessment to detect HIV-associated neurocognitive disorder: the challenge and need for culturally valid screening tests in South Africa. The clinical neuropsychologist, 27(3), 437-427. doi: 10.1080/13854046.2012.759627

Rodrigues, J. de C., Pawbaixaski, J., Zibetti, M. R., Fonseca, R. P., \& Parente, M. A. de M. P. (2011). Avaliação de apraxias em pacientes com lesão cerebrovascular no hemisfério esquerdo. Psicologia teoria e prática,13(2), 209-220. Recuperado de http://pepsic.bvsalud.org/pdf/ $\mathrm{ptp} / \mathrm{v} 13 \mathrm{n} 2 / \mathrm{v} 13 \mathrm{n} 2 \mathrm{a} 16 . \mathrm{pdf}$

Rosenstein, L. D. (1999). Visuoconstructional drawing ability in the differential diagnosis of neurologic compromise versus depression. Archives of clinical neuropsychology, 14(4), 359-372. doi: 10.1016/S0887-6177(98)00026-2

Rossetti, H. C., Lacritz, L. H., Cullum, C. M., \& Weiner, M. F. (2011). Normative data for the Montreal Cognitive Assessment (MoCA) in a population-based sample. Neurology, 77(13), 1272-1275. doi: 10.1212/WNL.0b013e318230208a

Royall, D. R., Chiodo, L. K., \& Polk, M. J. (2005). An empiric approach to level of care determinations: the importance of executive measures. Journal of Gerontology, 60(8), 1059-1064. doi: 10.1093/gerona/60.8.1059

Salles, J. F., Bandeira, D. R., Trentini, C. M., Segabinazi, J. D., \& Hutz, C. S. (2015). Teste de Retenção Visual de Benton (BVRT). Editora Vetor: Porto Alegre.

Santana, I., Duro, D., Freitas, S., Alves, L., \& Simões, M. R. (2013). The clock drawing test: Portuguese norms, by age and education, for three different scoring systems. Archives of Clinical Neuropsychology, 28(4), 375-387. doi: 10.1093/arclin/act016

Segabinazi, J. D., Duarte Jr, S., Salles, J. F. de, Bandeira, D. R., Trentini, C. M., \& Hutz, C. S. (2013). Teste de Retenção Visual de Benton: Apresentação do manual brasileiro. Avaliação Psicológica, 12(3), 429-432. Recuperado de http://pepsic.bvsalud.org/pdf/avp/v12n3/ v12n3a18.pdf

Shulman, K. (2000). Clock-drawing: Is it the ideal cognitive screening test? International journal of geriatric psychiatric, 15(6), 548-561. doi.org/10.1002/1099-1166(200006)15:6<548::AID-GPS242>3.0.CO;2-U

Taylor, L. B. (1969). Localization of cerebral lesions by psychological testing. Clinical and Neurosurgery, 16, 269-287. doi:10.1093/ neurosurgery/16.CN_suppl_1.269

Yesavage, J. A., Brink, T. L., Rose, T. L., Lum, O., Huang, V., Adey, M., \& Leirer, V. O. (1982). Development and validation of a geriatric depression screening scale: a preliminary report. Journal of Psychiatric Research, 17(1), 37-49. doi: 10.1016/0022-3956(82)90033-4

recebido em março de 2017 aprovado em junho de 2018

\section{Sobre as autoras}

Maila Rossato Holz é aluna de doutorado em Psicologia pela Pontifícia Universidade Católica do Rio Grande do Sul (PUCRS).

Renata Kochhann é aluna de pós-doutorado em Psicologia na PUCRS e pesquisadora no Hospital Moinhos de Vento.

Jerusa Fumagalli de Salles é professora da Universidade Federal do Rio Grande do Sul (UFRGS) no Instituto de Psicologia e no Programa de Pós-graduação (PPG) em Psicologia da UFRGS e bolsista de produtividade CNPq - Nível 1D.

Maria Alice de Mattos Pimenta é professora aposentada, visitante sênior do programa CAPES e colaboradora no Núcleo de Neuropsicolinguística do PPG em Psicologia da UFRGS.

Rochele Paz Fonseca é professora titular da faculdade de Psicologia e no PPG em Psicologia da PUCRS. É bolsista de produtividade CNPq - Nível 1D. 